\title{
INFINITESIMALLY RIGID POLYHEDRA. II: MODIFIED SPHERICAL FRAMEWORKS
}

\author{
WALTER WHITELEY
}

\begin{abstract}
In the first paper, Alexandrov's Theorem was studied, and extended, to show that convex polyhedra form statically rigid frameworks in space, when built with plane-rigid faces. This second paper studies two modifications of these polyhedral frameworks: (i) block polyhedral frameworks, with some discs as open holes, other discs as space-rigid blocks, and the remaining faces plane-rigid; and (ii) extended polyhedral frameworks, with individually added bars (shafts) and selected edges removed. Inductive methods are developed to show the static rigidity of particular patterns of holes and blocks and of extensions, in general realizations of the polyhedron. The methods are based on proof techniques for Steinitz's Theorem, and a related coordinatization of the proper realizations of a 3-connected spherical polyhedron. Sample results show that: (a) a single $k$-gonal block and a $k$-gonal hole yield static rigidity if and only if the block and hole are $k$-connected in a vertex sense; and (b) a 4-connected triangulated sphere, with one added bar, is a statically rigid circuit (removing any one bar leaves a minimal statically rigid framework). The results are also interpreted as a description of which dihedral angles in a triangulated sphere will flex when one bar is removed.
\end{abstract}

1. Introduction. In our first paper [25] (hereafter referred to as I), we extended Cauchy's original theorem on the rigidity of triangulated convex polyhedra into an analysis of the infinitesimal (or equivalently, the static rigidity) of general convex polyhedra built with joints at the vertices, bars along the edges, and bars triangulating the faces. This analysis showed that plane-rigid faces fit together to give space-rigid structures, and was extended to cover tensegrity frameworks, and even $d$-dimensional polytopes. The results applied to built structures such as the standard geodesic domes, as well as less standard space frames such as polyhedra with cabled faces [12].

In this second paper, we build on Steinitz's Theorem (the realizability of 3connected planar graphs as convex polyhedra), to analyze modified polyhedral frameworks, in which certain discs become open holes (no bars), other discs are blocked (a space-rigid framework) and the remaining faces are triangulated (a planerigid framework). As a basic theorem, illustrating the methods developed, we show

Received by the editors August 9, 1985. The results were presented at the AMS meeting, Amherst, Massachusetts, October 1981, in the special session on Discrete Geometry and its Applications.

1980 Mathematics Subject Classification (1985 Revision). Primary 51M20, 52A25, 70C99; Secondary 05C10, 70B99, 73K99.

Key words and phrases. Infinitesimal rigidity, static rigidity, generic rigidity, polyhedral framework, 4-connected graph, Steinitz's Theorem.

Work supported, in part, by grants from N.S.E.R.C. (Canada) and F.C.A.R. (Quebec), and a visiting appointment at McGill University. 
that a 3-connected spherical polyhedron, with one $k$-gonal hole and one $k$-gonal block, is statically rigid in general realizations if and only if the hole and the block are $k$-connected in a vertex sense. In this simple form the result applies to triangulated cylinders, with two holes, which are to be rigidified by fastening one end to the ground [20]. The methods developed for block polyhedral frameworks also apply to general geodesic domes which have been grounded (a block) and then had bars removed for windows and doors.

The same theorem answers a question of Kuiper [14 Remark 9]: when a single bar is omitted from a triangulated sphere - which dihedral angles begin to flex? The theorem translates to say: the removed bar makes a dihedral angle flex, in general realizations, if and only if the quadrilateral hole is 4-connected to the quadrilateral of the angle. In particular, as suggested by Kuiper, in a 4-connected sphere the omission of one bar makes all dihedral angles flex.

In $\S 5$, we switch perspective away from the discs of faces, and add extra bars to a spherical polyhedron built with triangulated faces - the extended polyhedral frameworks. In particular we show that any 4-connected triangulated sphere, with one added edge between nonadjacent vertices, forms a statically rigid circuit in general realizations. A statically rigid circuit is a framework for which the omission of any one bar leaves a minimal statically rigid spatial framework. In practical terms it is optimally over-braced, since the failure of any one member will not cause a collapse. Such circuits also play a central role as minimal statically rigid tensegrity frameworks [17]. Thus a 4-connected sphere, with an added shaft, can be built with each member replaced by either a cable (a pure tension member) or a strut (a pure compression member).

To obtain these rigidity results, we must explore some basic facts about 3connected spherical polyhedra. In particular, we build on several inductive techniques based on the proof of Steinitz's Theorem:

(i) Given an abstract 3-connected spherical polyhedron, and a proper subnet (a smaller polyhedron with some of the vertices, with paths as edges, and the induced faces), there is a construction sequence $P_{1}, P_{2}, \ldots, P_{n}=P$ in which $P_{i+1}$ comes from $P_{i}$ by a single step of edge splitting, face splitting, or inserting a 3 -valent vertex over a triangle [Theorem 2.1].

(ii) Given an abstract 3-connected spherical polyhedron, the faces and vertices form a Steinitz list, in which each vertex (face) appears at a point where it is incident with 3 or less faces (vertices) in the list $[\mathbf{4}, \mathbf{1 6}]$.

The construction sequence is first used to show that the polyhedron $P$ has a proper realization (no extra collinearity of vertices and faces) near any proper realization of the proper subnet (a variant of Steinitz's Theorem). The Steinitz list is used in this induction to realize face splits. This list is also used to generate a coordinate system for the proper realizations, in which the statically rigid block polyhedral frameworks form an open dense subset. These two results are combined with inductive techniques for spatial frameworks to show:

THEOREM 3.14. If an abstract block and hole polyhedron contains a proper subnet (containing all the blocks and holes) which has a statically rigid block polyhedral framework in some realization, then almost all realizations of the final polyhedron as block polyhedral frameworks are statically rigid. 
The analysis of block polyhedral frameworks now reduces to finding appropriate proper subnets which can easily be built as statically rigid frameworks, and can be shown to exist from simple connectivity assumptions about the polyhedron. For this we use Dirac's extension of Menger's Theorem $[\mathbf{8}, \mathbf{1 8}]$, which translates vertex connectivity into the existence of specified vertex-disjoint paths. The increasing complexity of such arguments is the only limit to the structures which we can analyze.

We need these special techniques for frameworks built around spherical polyhedra because the general problem of characterizing the graphs of statically rigid frameworks in space is unsolved $[\mathbf{1 0}, \mathbf{2 1}, \mathbf{2 2}]$. The modified polyhedral frameworks studied here represent an initial effort to fill the gap between the well-understood triangulated spheres and the difficult general frameworks.

ACKNOWLEDGEMENTS. This work grows on the ground prepared by joint work in the Structural Topology Research Group at the University of Montréal, involving, in particular, Janos Baracs and Henry Crapo. Many of the seed questions about polyhedral frameworks were planted by Branko Grünbaum [12] and Robert Connelly $[\mathbf{5}, \mathbf{6}]$, and Robert Connelly has provided stimulation throughout the years during which these results have matured.

2. Polyhedral inductions. As we mentioned in the Introduction, we will inductively build up the final polyhedron, with designated discs, from a selected initial polyhedron. The steps we choose are restricted by the requirement that (i) each intermediate polyhedron must be realized nearby as a proper spatial polyhedron, and (ii) each intermediate structure must be statically rigid in this realization. On the other hand, the steps must be sufficient to reach the desired class of final polyhedra.

A number of the methods are refinements of Steinitz's Theorem, and of some inductive methods developed for its proof.

THEOREM (STEINITZ $[\mathbf{4}, \mathbf{1 1}, \mathbf{1 6}, \mathbf{1 9}]$ ). A graph can be realized as the edge skeleton of a convex spherical polyhedron if and only if it is a 3-connected planar graph.

2.1. Abstract polyhedra and subnets. We begin with a precise description of the graphs involved. Our attention will be focussed on spherical polyhedra which can be statically rigid. Accordingly we begin with planar graphs.

DEFINITION 2.1. A 3-connected planar graph $G=(V, E)$ is a finite set of vertices $V$ and edges $E$ (unordered pairs of distinct vertices) such that

(i) the graph can be drawn with points in the plane, simple smooth curves for edges, and no edges crossing (i.e. it is planar);

(ii) if any two vertices (and their adjacent edges) are deleted, the graph remains connected: for any other two vertices there is a path of edges $\left(u_{1}, v_{1}\right),\left(v_{1}, v_{2}\right), \ldots$, $\left(v_{n}, u_{2}\right)$ in the remaining graph (i.e. it is 3-connected in a vertex sense).

Any planar drawing of a planar graph defines a set of faces: deleting the points and curves leaves topological discs (faces) each bounded by a cycle of vertices and edges (the face polygon) $v_{1} v_{2} \cdots v_{n} v_{1}$ as well as the exterior unbounded region which also forms a face. If the graph is 3-connected, this face structure is unique $[30,31]$. (In fact a face is then any simple cycle of vertices and edges whose removal does not disconnect the edges of the graph.) 


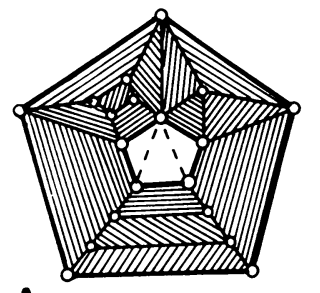

A

B

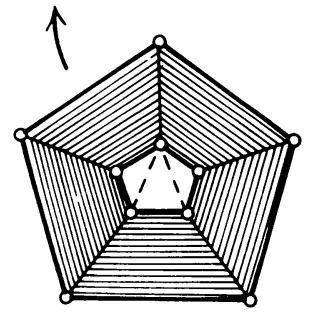

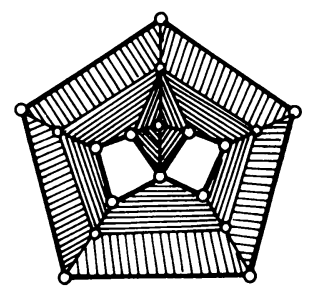

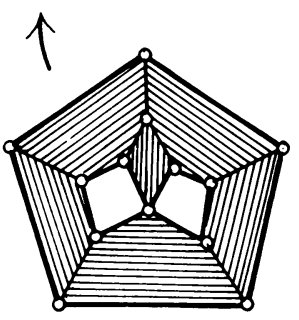

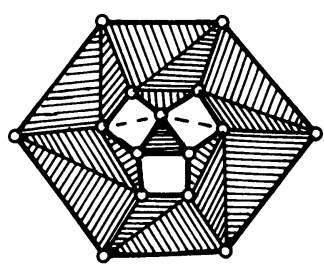

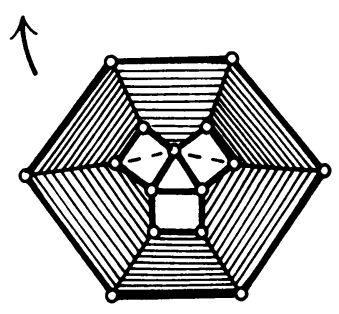

FIGURE 2.1

DEFINITION 2.2. An abstract 3-connected spherical polyhedron $(V, F ; I)$ (or polyhedron for short) is the set of vertices and faces of a 3-connected planar graph, such that $\left(v_{i}, f_{j}\right) \in I$ if and only if vertex $v_{i}$ is in the cycle of face $f_{j}$.

REMARK. This is equivalent to other standard definitions of a 3-connected spherical polyhedron. In particular it includes the usual properties of an abstract polyhedron [26]:

(i) The faces $f_{i}$ in $F$ incident with a vertex $j$ in $V$ form a cycle of distinct faces $f_{j}^{1}, f_{j}^{2}, \ldots, f_{j}^{s}(s \geq 3)$.

(ii) The vertices $v_{j}$ in $V$ incident with a face $i$ in $F$ form a cycle of distinct vertices $v_{1}^{i}, v_{2}^{i}, \ldots, v_{t}^{i}(t \geq 3)$.

(iii) An edge is an adjacent pair of vertices in some face cycle. Each pair of vertices $v_{j}, v_{k}$ adjacent in a cycle for $f^{h}$ are adjacent in exactly one other face $f^{i}$, and this pair of faces is adjacent in the two cycles of these vertices.

The fact that an abstract polyhedron is spherical can also be given by means of the Euler characteristic: $X=v-e+f$ (where $v, e$, and $f$ represent the size of $V, E$, and $F$ respectively). For a general connected abstract polyhedron (a set of vertices and faces with the required cycle structure), $X=2$ is equivalent to the topological fact that the polyhedron is spherical.

In Chapter 3 we will select certain special pieces of this polyhedron to be open holes, others to be space-rigid blocks, and the remaining faces will be triangulated plane polygons. We need some additional vocabulary for these special regions. Each simple polygon (cycle of distinct vertices and edges) separates the spherical polyhedron into two components which are discs. A simple disc is such a component containing no vertices, and the edges of this polygon are boundary edges. Two simple discs are disjoint if they share at most one edge (a boundary edge) and no faces.

DEFINITION 2.3. A bounded 3-connected spherical polyhedron (or bounded polyhedron for short) is a 3-connected spherical polyhedron with a set $D$ of simple, pairwise-disjoint discs. 
The disc set is the set of vertices, edges and faces in the discs, and the boundary edges of the polyhedron are the boundary edges of the discs. The vertices, edges and faces not in the disc set are regular.

In Figure 2.1 A, we show some bounded polyhedra, with the discs shown blank, and the regular faces shaded. In each case the enclosing polygon is a disc of $D$, the boundary edges are heavy, and edges interior to any disc are shown as dotted lines. In the inductions we will use substructures of the polyhedron, whose edges are paths of edges in $P$ (Figure $2.1 \mathrm{~B}$ ).

DEFINITION 2.4. A simple polygonal path in a polyhedron $P$ is a sequence of distinct vertices $v_{1} v_{2} \cdots v_{n}$ such that $v_{i}, v_{i+1}$ is an edge of $P$. for all $i$. This is called a path for short and $v_{2}, \ldots, v_{n-1}$ are interior vertices of the path, while $v_{1}, v_{n}$ are the terminal vertices.

Two paths are disjoint if any common vertices are terminal in each path.

DEFINITION 2.5. A subnet $P^{\prime}$ of a polyhedron $P$ is a set $E^{\prime}$ of pairwise disjoint paths in $P$, a set $V^{\prime}$ of all terminal vertices of these paths, making the graph $\left(V^{\prime}, E^{\prime}\right)$ at least 2-connected with no multiple edges, and a set $F^{\prime}$ of faces cut from $P$ by the paths $E^{\prime}$.

A path vertex of $P^{\prime}$ is an interior vertex of a path $e^{\prime}$ in $E^{\prime}$.

A skeletal vertex is a 2-valent vertex of $P^{\prime}$.

A natural vertex is a vertex of $P^{\prime}$ which is 3 or more valent.

A natural edge is a pair of natural vertices $s, t$ joined by a path in $P^{\prime}: s=$ $v_{1}, v_{2}, \ldots, v_{n}=t$, where $v_{i}$ are skeletal vertices, $1<i<n$.

DEFINITION 2.6. A proper subnet $P^{\prime}$ of a bounded polyhedron $(P, D)$ is a subnet $P^{\prime}$ such that:

(i) $P^{\prime}$ contains all vertices, edges, and faces of the disc set $D$;

(ii) the natural vertices and edges of $P^{\prime}$ form a 3-connected planar graph.

THEOREM 2.1. Given any bounded polyhedron $(P, D)$ and proper subnet $P^{\prime}$, there is a sequence of proper subnets $P^{\prime}=P_{j}=P_{1}, P_{2}, \ldots, P_{n}=P$ such that $P_{i+1}$ comes from $P_{i}$ by

Step (a) replacing a path in $P_{i}$ of one regular edge in $P$ by a longer path in $G$ joining the same terminal vertices, pairwise disjoint from the paths in $P_{i}$ (edge stretching);

Step (b) dividing a path in $P_{i}$ into two paths at a path vertex, which becomes a new skeletal vertex of $P_{i+1}$ (edge splitting);

Step (c) inserting a new path across a regular face, disjoint from the paths in $P_{i}$, such that the terminal vertices of the path are skeletal or natural vertices of $P_{i}$, and they are separated by the remaining natural vertices of $P_{i}$ (face splitting);

Step (d) inserting a 3-valent vertex attached to three vertices $s, t, u$, such that all 3 edges among $s, t, u$ are boundary edges (pointing a boundary triangle).

PrOOF. Each step of the construction increases the sum of the number of vertices in $P_{i}$ plus the number of edges of $P$ in paths of $P_{i}$. If we have such a $P_{i} \neq P$, we will show how to find the next subnet $P_{i+1}$. Since the number of vertices and edges in $P$ is finite, the construction will then terminate with $P$.

If $P_{i}$ has a path vertex $v$, we break the corresponding path at $v$ and create $P_{i+1}$ with an additional skeletal vertex using Step (a). Since $P_{i+1}$ has the same natural vertices and edges, it clearly is a proper subnet containing the disc set. 

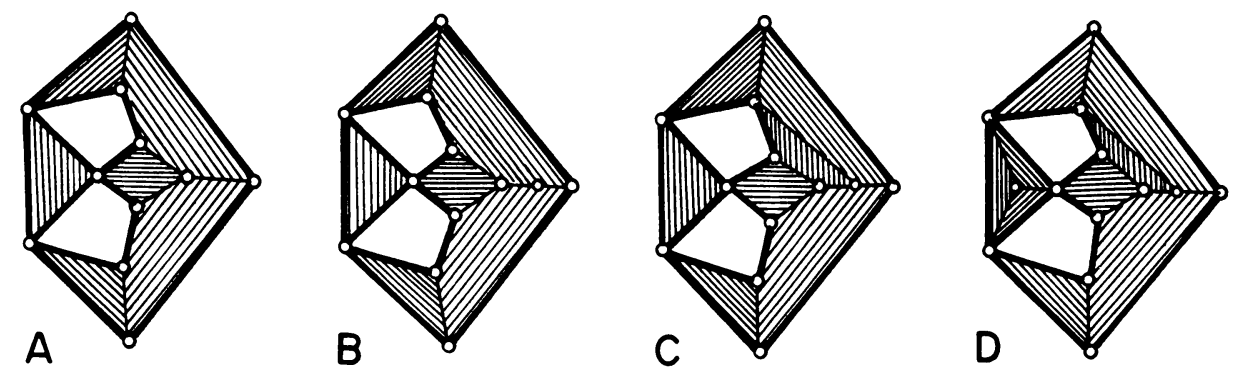

FIGURE 2.2

Assume that $P_{i}$ has no path vertices, but has a skeletal vertex $r$ on the natural edge $u, v$. Since $P$ is 3-connected, there is a path in $P$ joining $r$ to some other natural vertex $w$, which avoids $u$ and $v$. Let $s$ be the last vertex of this path which is on $u v$, and let $t$ be the next vertex of this path which is a vertex of $P_{i}$. The path $s t$ is disjoint from the paths of $P_{i}$, so we can add this path as a face split (Step (c)). Clearly this $P_{i+1}$ contains all the disc set. The new graph of natural vertices and natural edges is still 3-connected: any two vertices which disconnect the graph would either create two components in $P_{i}$, which is impossible, or create a component with only $s, t$, or both. A simple inspection shows that these sets have sufficient connections to prevent this.

Assume that there are no path or skeletal vertices in $P_{i}$, but there is a vertex $r$ of $P$ not in $P_{i}$. Since $P$ is 3-connected, there are 3 vertex-disjoint paths from $r$ to $P_{i}$, ending in vertices $s, t, u$. If $s, t, u$, is a triangle of boundary edges, then we point this boundary triangle, using Step (d). Otherwise some pair $s, t$ is not a boundary edge, and we add $s, r, t$ as a path (new edge) splitting a face by Step (c) to create $P_{i+1}$. In either case, $P_{i+1}$ contains the disc set, and has the same natural vertices, as well as more natural edges. Therefore it is the required proper subnet.

Finally, if all vertices of $P$ are present in $P_{i}$, and an edge is missing, we add this edge as a face split, to create an appropriate $P_{i+1}$.

REMARK 1. If we insist that all paths chosen, including those in $P^{\prime}$ which are not in the disc set, are maximal in terms of the number of path vertices, then the edge stretching of Step (a) would be unnecessary, following the pattern of Barnette and Grünbaum [4]. However, it will be convenient to choose $P_{i}$ without this extra concern.

REMARK 2. While Steps (a) and (b) do not change the faces of $P_{i}$, Step (c) clearly splits a regular face into 2 regular faces, and Step (d) breaks a regular triangle into three regular triangles. In the entire construction, the disc set of vertices, edges and faces is never changed.

For later reference we call the sequence created in this theorem a construction sequence for $P$ from $P^{\prime}$. Figure 2.2 gives the construction sequence for a polyhedron (D) from the proper subnet (A), illustrating Steps (b), (c), and (d) in the corresponding parts of the figure. The natural vertices and edges of any $P_{i}$ form a bounded polyhedron. When we add the skeletal vertices of $P_{i}$ this gives an abstract refined spherical polyhedron. These differ from the original polyhedra because we allow skeletal vertices which have a cycle of only 2 faces. As a shorthand notation we will also call this refined polyhedron $P_{i}$. 
2.2. Proper realizations. Having constructed a sequence of abstract refined polyhedra, we want to construct a sequence of spatial polyhedra with all faces plane, and no unnecessary collinearity of faces or vertices.

DEFINITION 2.5. A proper realization of an abstract spherical polyhedron is an assignment of planes to the faces and points to the vertices such that

(i) if $f_{i}$ is incident to the vertex $v_{i}$, then the plane contains the corresponding point;

(ii) no three vertices of a face are collinear;

(iii) no three faces at a vertex are collinear.

Note that this implies no adjacent faces are coplanar.

A proper realization of a refined abstract spherical polyhedron is a proper realization of the natural polyhedron, with skeletal vertices on the lines of their natural edges, chosen so that three vertices of a face are collinear only if they share a natural edge.

The inductive construction of the proper realizations is based on a second technique of Steinitz. We give the classical result without proof $[\mathbf{4}, \mathbf{1 6}, \mathbf{1 9}]$.

THEOREM 2.2 (STEINITZ). All vertices and faces of an abstract 3-connected spherical polyhedron can be arranged in a sequence, beginning with any chosen edge, $f_{0}, f_{1}, v_{0}, v_{1}, \ldots, f_{i}, \ldots, v_{j}, \ldots$ such that each vertex and face is incident with at most 3 previous elements in the list.

It is a simple matter to extend this list to include the skeletal vertices of a refined polyhedron by adding them to the end of the list.

COROLLARY 2.3. All vertices and faces of an abstract refined 3-connected spherical polyhedron can be arranged in a sequence, beginning with any chosen natural edge $f_{0}, f_{1}, v_{0}, v_{1}, \ldots, f_{i}, \ldots, v_{j}, \ldots$ such that each vertex and face is incident with at most 3 previous elements in the list, and all skeletal vertices are placed at the high end.

We call this list a Steinitz list for the polyhedron. We now have a variant of Steinitz's Theorem.

THEOREM 2.4. Given a construction sequence $P^{\prime}=P_{1}, \ldots, P_{n}=P$ for an abstract spherical polyhedron $P$, such that $P^{\prime}$ has a proper realization then each refined abstract spherical polyhedron $P_{i}$ has a proper realization.

PROOF. We will construct the desired realizations by induction. We assume that $P_{i}$ has a proper realization, and construct $P_{i+1}$ for each of the possible steps.

Step (a). This causes no change in the vertices or faces of $P_{i}$, so we have the required realization.

Step (b). For the new skeletal vertex we choose a general point on the line segment of the original edge. This clearly gives a proper realization of $P_{i+1}$.

Step (c). Since $P_{i+1}$ is an abstract refined 3-connected spherical polyhedron, we arrange the faces and vertices in a sequence beginning with the new natural edge to be added. This edge will split a face of $P_{i}$ into 2 faces. We place one of the new faces as the same plane as the original in $P_{i}$, and turn the second plane slightly through the points of the common vertices in the realization $P_{i}$. We now move up the sequence, choosing planes and points as permitted by the maximum of 3 
incidences. When there are fewer than 3 incidences, we keep the choice close to the assignment in $P_{i}$. In the end we obtain a realization of $P_{i+1}$ which is as close as we want to that for $P_{i}$. Since the points and planes were distinct in the realization of $P_{i}$, they are still distinct in the new realization, and it is proper.

Step (d). The polyhedron $P_{i}$ is realized with a noncollinear triangle $s, t, u$. If we place $r$ off all face planes, we obtain a proper realization of $P_{i+1}$.

REMARK. For our later work on statics we note that in the face splitting of Step (c) the points in the new realization of $P_{i+1}$ (i.e. a point in $R^{3 v}$ ) can be made arbitrarily close to the points chosen for $P_{i}$.

2.3 Connectivity and paths. We also need a basic correspondence between connectivity of a graph, expressed in terms of separation by removing vertices (cut sets) and connectivity expressed in terms of vertex disjoint paths (flows). The following result is a variant of the Ford-Fulkerson max. flow = min. cut theorem, but it was first given, independently, by Dirac.

THEOREM 2.5 (DIRAC $[8,18]$ ). Given a graph $G$ and two sets of vertices $A=\left\{a_{1}, \ldots, a_{k}\right\}$ and $B=\left\{b_{1}, \ldots, b_{m}\right\}$ such that no vertex of $A$ can be separated from a vertex of $B$ by a set of fewer than $n$ vertices, then there exist $n$ paths in $G$ such that

(i) $c_{i}$ of the paths start at $a_{i}$,

(ii) $d_{i}$ of the paths end at $b_{j}$,

(iii) the paths are disjoint except at terminal vertices, provided $c_{1}+\cdots+c_{k}=d_{1}+\cdots+d_{m}=n$.

We illustrate the use of this theorem with the simplest result on proper subnets.

THEOREM 2.6 (STEINITZ [4]). Every abstract 3-connected spherical polyhedron contains a tetrahedral subnet (a subnet of 4 vertices and 6 paths joining all pairs).

PROOF. Take any face cycle $r_{1} \cdots r_{n}$ and any vertex $s$ not on this face. By the 3-connectivity, and Dirac's Theorem, there are 3 vertex-disjoint paths from $s$ to this polygon. If $t_{1}, t_{2}$, and $t_{3}$ are the first polygon-points on these paths, then the polygon, as a triangle $t_{1}, \ldots, t_{2}, \ldots, t_{3}, \ldots, t_{1}$ and the three paths $s, \ldots, t_{1}$, $s, \ldots, t_{2}$, and $s, \ldots, t_{3}$ form a tetrahedral subnet with vertices $s, t_{1}, t_{2}, t_{3}$.

The tetrahedron has proper, convex realizations. If we apply Theorem 2.4 to an arbitrary abstract 3-connected spherical polyhedron, starting with such a realization of the tetrahedral subnet, we have one of the standard proofs of Steinitz's Theorem [4]. We simply take care to bend the faces of Step (c) to preserve the convexity.

\section{Statics of polyhedral frameworks.}

3.1 Statics of general frameworks. We are primarily interested in spatial frameworks, but we will need certain properties of plane frameworks, since they will form the faces. The basic definitions and results were presented in the first paper, and we briefly summarize them here.

DEFINITION 3.1. A bar and joint framework in 3-space is a graph $G=(V, E)$, with $v$ vertices and $e$ edges, and a mapping $Q: V \rightarrow R^{3}$ such that $Q(a) \neq Q(b)$ if $(a, b) \in E$. 
As a convention, we write the framework as $F=(G, Q)$ and the joints $Q(i)$ as $q_{i}$, and sometimes as $i$, when the framework is clear. In a framework we also speak of the bar $(i, j)$ or $q_{i} q_{j}$ and abuse notation to write edges and bars as $i j$.

DEFINITION 3.2. An equilibrium load on a framework $F=(G, Q)$ is an assignment $L$ of 3 -vectors to the vertices $\left(L_{1}, \ldots, L_{v}\right)$ such that

(i) $\sum L_{i}=0$ (sum over all vertices) and (ii) for each pair of indices $1 \leq h<k \leq 3$, $\sum\left[\left(L_{i}\right)_{h}\left(q_{i}\right)_{k}-\left(L_{i}\right)_{k}\left(q_{i}\right)_{h}\right]=0$ (sum over all vertices) where $(x)_{k}$ denotes the $k$ th component of the vector $x$.

For an edge $(i, j), i<j$, the edge load is $L_{i j}=\left(0, \ldots, q_{i}-q_{j}, 0, \ldots, 0, q_{j}-\right.$ $\left.q_{i}, 0, \ldots, 0\right)$ with the 3 -vector $q_{i}-q_{j}$ in the 3 columns for vertex $i, q_{j}-q_{i}$ in the columns for $j$ and zero elsewhere.

A resolution of the load $L$ by a framework is an assignment of scalars $\lambda_{i j}$ to the edges $i j E$ such that $\sum \lambda_{i j} L_{i j}+L=0$ (sum over all edges).

A bar and joint framework in 3-space is statically rigid if and only if every equilibrium load in 3-space has a resolution.

A bar and joint framework in a plane is plane-statically rigid if and only if every equilibrium load in the plane has a resolution.

To show that a framework is statically rigid we must generate the vector space of all equilibrium loads. Following the usual patterns in linear algebra, we will count the dimension, and then produce an independent set of this size-a basis. For bar and joint frameworks, such a basis - a minimal statically rigid framework on a set of joints-is called isostatic. A dependence among the edge loads is called a nontrivial stress. Thus a stress is a set of scalars $\lambda_{i j}$ such that $\sum \lambda_{i j} L_{i j}=0$, and it is nontrivial if some $\lambda_{i j} \neq 0$. If a framework has only the trivial stress it is independent.

We recall the following basic facts (Corollaries I.2.2 and I.2.3).

THEOREM 3.1. Given $a$ bar and joint framework $F=(G, Q)$ in 3-space with $v \geq 3$, the following are equivalent:

(i) $F$ is isostatic;

(ii) $e=3 v-6$ and the framework is independent;

(iii) $e=3 v-6$ and the framework is statically rigid.

THEOREM 3.2. If a bar and joint framework is isostatic in 3-space then adding or removing a 3-valent joint, with the bars not coplanar, creates a new isostatic framework.

If a bar and joint framework is plane-isostatic then adding or removing a 2-valent joint, with the bars not collinear, creates a new plane-isostatic framework.

An isostatic framework is a basis for the subspace of equilibrium loads on the vertices. From linear algebra, we know that one such basis can be replaced by another, even within a larger set, without changing the space which is generated, or alternating properties such as independence. We will use the following broad substitution principle based on this observation (Theorem I.2.6).

THEOREM 3.3. (a) If a framework is isostatic (statically rigid) in 3-space, with the subframework on $k$ joints statically rigid in the affine plane of its joints, and this subframework is replaced by another subframework on the same joints isostatic in the same plane, then the modified framework is isostatic (statically rigid) in 3-space. 
A

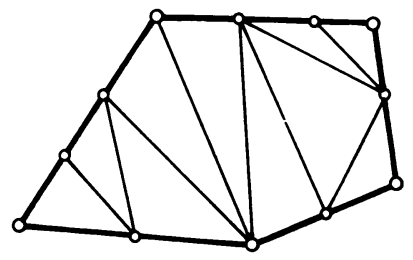

B

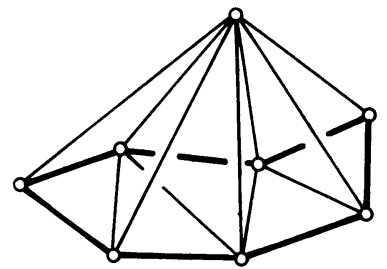

FIGURE 3.1

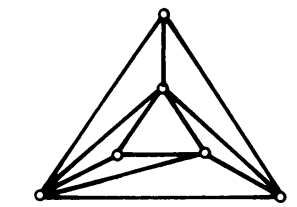

A

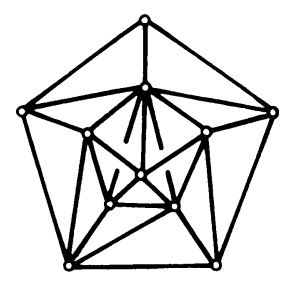

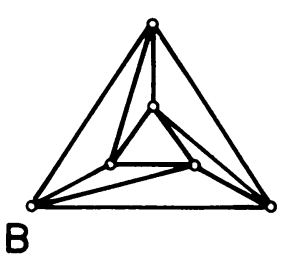

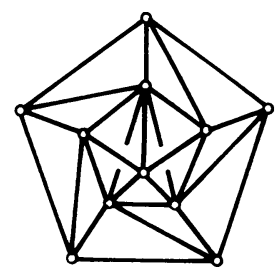

FIGURE 3.2

(b) If a framework is isostatic (statically rigid) in 3-space, with the subframework on $k$ joints statically rigid in space, and this subframework is replaced by another subframework isostatic on the same joints, then the modified framework is isostatic (statically rigid) in 3-space.

We will need to have some appropriate plane-rigid bar and joint frameworks for the regular faces of the polyhedron. These will be triangulated proper plane polygons - a cycle of joints and bars in a plane with no 3 points collinear, which are triangulated with additional bars. These plane frameworks satisfy $e=2 v-3$, and can be built up from a single bar by a sequence of adding 2-valent noncollinear joints. In addition, we will want to add skeletal vertices, and refine the proper plane polygon. If we use any triangulation with no triangle formed from vertices of a natural edge, and insist that all other triples are not collinear, we have refined triangulated proper plane polygons (Figure $3.1 \mathrm{~A}$ ). The following simple result applies (Proposition I.2.5).

PROPOSITION 3.4. A triangulated polygon, with no triangle collinear, is planeisostatic.

Any triangulated proper plane polygon, and any refined triangulated proper plane polygon, are plane-isostatic.

Similarly, we can take a spatial polygon (no 3 points collinear, not necessarily coplanar) which is triangulated by bars. If we add one extra joint (not coplanar with any triple of the previous joints), and connect it to all the other joints, we create a proper blocked polygon (Figure $3.1 \mathrm{~B}$ ). Since this framework can be built from a triangle by adding a sequence of noncoplanar 3-valent joints, we have the following result.

PROPOSITION 3.5. A proper blocked polygon is an isostatic bar and joint framework in space.

This framework is an example of a simple framework - it can be built from a triangle by a sequence of 3-valent joints. Other examples of simple frameworks, which 
we will use later, are shown in Figure 3.2 A. Of course most isostatic frameworks are not simple (Figure $3.2 \mathrm{~B}$ ) and these complex frameworks present the essential problems.

\subsection{Infinitesimal motions and generic properties.}

DEFINITION 3.6. An infinitesimal motion of the framework $F=(G, Q)$ is an assignment of 3-vectors to the vertices $M=\left(m_{1}, \ldots, m_{v}\right)$, the velocities, such that for each edge $i j \in E,\left(q_{i}-q_{j}\right) \cdot\left(m_{i}-m_{j}\right)=0$.

These equations form the rows of the rigidity matrix of the framework. These rows are also the edge loads defined above. There will always be certain trivial infinitesimal motions - such as the translations $(m, \ldots, m)$ for any vector $m$ and the rotations $M_{h k}$ with $1 \leq h<k \leq 3, m_{i}=\left(0, \ldots, 0,\left(q_{i}\right)_{k}, 0, \ldots, 0,\left(q_{i}\right)_{h}, 0, \ldots, 0\right)$. These rotations and translations generate the space of rigid motions. An internal motion is an infinitesimal motion which is not a rigid motion.

DEFINITION 3.7. A bar and joint framework is infinitesimally rigid if and only if every infinitesimal motion is a rigid motion.

There is a correspondence between these rigid motions and the equilibrium equations of statics. From this observation, and the fact that row rank = column rank in the rigidity matrix, the following fundamental facts follow [17].

THEOREM 3.7. A bar and joint framework does not resolve the load $L_{s t}$ if and only if there is an infinitesimal motion $M$ such that $\left(m_{s}-m_{t}\right) \cdot\left(q_{s}-q_{t}\right) \neq 0$. A bar and joint framework in 3-space is infinitesimally rigid if and only if it is statically rigid.

A given graph has a set of realizations $Q \in R^{3 v}$, with $q_{i} \neq q_{j}$ if $i j \in E$. The rank of $R(G, q)$ is determined by minors being zero-i.e. polynomial equations in the coordinates of $Q$ [23]. When some points make these polynomials nonzero, then almost all values will. (These values form an open dense subset of $R^{3 v}$.) We can think of choosing a set of variables for the $\left(q_{i}\right)_{j}$ (or more exactly a set of algebraically independent real numbers over the rationals, forming a general point) and use these to find the common or "generic" properties.

It is known that if a graph $G$ has a generic property in 3-space, then the realizations in 3-space with this property form an open dense subset of $R^{3 v}$. It is also known that if almost all realizations of a graph are even finitely rigid (lack finite motions) then almost all realizations are statically, or equivalently infinitesimally rigid [2]. This justifies the use of the term generically 3-rigid. Conversely, if a framework has an infinitesimal internal motion in all realizations, then almost all realizations have finite, nonrigid motions.

PROPOSITION 3.8. A graph $G$ is generically 3-isostatic (3-rigid) if and only if there is at least one framework realizing $G$ in 3-space which is isostatic (statically rigid).

\subsection{Polyhedral frameworks.}

DEFINITION 3.11. A polyhedral framework is a realization of a proper polyhedron $((V, F ; I), Q)$ as a bar and joint framework in projective 3 -space with

(i) a joint for each vertex;

(ii) a bar for each edge of the polyhedron;

(iii) additional bars for each face to triangulate the face. 
As we saw above, the statically rigid frameworks form an open set in the space $R^{3 v}$ of all realizations, and if this set is nonempty, it is dense in the space (the rigidity almost always occurs). We would like similar results for the class of polyhedral frameworks - but we have the basic problem that there is no simple euclidean space of realizations. The realizations of a polyhedron form an algebraic variety in $R^{3 v+4 f}$ defined by the equations $a_{1} q_{1}+a_{2} q_{2}+a_{3} q_{3}+a_{4}=0$ for each vertex $\left(q_{1}, q_{2}, q_{3}\right)$ incident to the plane with coordinates $\left(a_{1}, a_{2}, a_{3}, a_{4}\right)$. If two polyhedral frameworks are close in this variety, then they are also close as bar and joint frameworks. This gives the first property we need.

THEOREM 3.10. For a given polyhedron, the set of statically rigid polyhedral frameworks is an open subset of the variety of all polyhedral frameworks.

It would be simple to show that the statically rigid frameworks are dense in the variety if the variety was irreducible. However, this is not true (see Remark 2). However, we can take the set of proper realizations (which is not a variety) and show by a direct coordinatization of the subset, that static rigidity either never occurs or almost always occurs. The key to our coordinate system is the Steinitz list of the vertices and faces.

THEOREM 3.11. The set of proper realizations of an abstract 3-connected spherical polyhedron is coordinatized by an open dense subset of $R^{c}$ where $c=$ $e+f+6$. The euclidean coordinates of the points and planes are rational functions of these choice coordinates.

If one proper polyhedral framework on $P$ is statically rigid, then the statically rigid realizations form an open dense subset in this coordinatization.

ProOF. Take a Steinitz list for the polyhedron, beginning at any edge. Make an arbitrary choice for the coordinates of the two planes (8 choices), ensuring they are distinct and not parallel. For each vertex of this edge, we must satisfy the 2 linear equations (they lie on the 2 planes) so we have a 1-parameter set of choices. These parameters are the next 2 coordinates, chosen to keep the points distinct. The remaining euclidean coordinates are found as rational functions of the coordinates of the planes and these parameters.

At each stage in the list, we have evolved a set of $3 v+4 f-i$ choice coordinates. The euclidean coordinates of all current planes and points are rational functions of these choice coordinates, and when these rational functions are defined, we have a proper realization. At the next step in the list we add a new vertex or face, incident with $j \leq 3$ previous elements of the list.

For a face, this gives $j \leq 3$ homogeneous linear equations in 4 unknowns, with the coefficients given as rational functions of the previous choices. Since, for a proper realization, these are independent, we have $4-j$ parameters to choose. The euclidean coordinates of the plane are then rational functions of these parameters and the coefficients and hence, rational functions of the choice coordinates. (We must also ensure that the plane is finite, and not $(0,0,0,1)$ but this is mererly another determinant condition to be avoided.)

Similarly, for a vertex we have $j$ linear equations in the 3 variables. Since there are proper realizations, these are, in general, independent and consistent. This leaves $3-j$ parameters to add to the choice coordinates, so that the solutions are rational functions of the choice coordinates. 
In both cases, we conclude that there are $3 v^{\prime}+4 f^{\prime}-i^{\prime}$ choice coordinates if we have $v^{\prime}, f^{\prime}$ and $i^{\prime}$ vertices, faces and incidences at this position in the list. By induction this will be true for the final list for the spherical polyhedron. However, by Euler's formula $v-e+f=2$. Each edge represents 4 incidences, and each incidence occurs in 2 edges, so $2 e=i$. By simple algebra this yields $3 v+4 f-i=e+f+6$, as required.

(ii) The static rigidity of any framework is characterized by a set of polynomial conditions in the euclidean coordinates of the vertices. If we replace these vertices by the rational functions of the choice coordinates, and clear fractions, we have a set of polynomial conditions in the choice coordinates. Since there is some statically rigid proper polyhedral framework, there is some choice which makes one of the polynomials nonzero. Therefore almost all values of the choice coordinates make this condition nonzero, and almost all proper realizations have statically rigid polyhedral frameworks.

REMARK 1. The choice coordinates also describe many improper realizations. The coordinates actually break down when either (i) the equations in some step are dependent, because a particular triple of vertices on a face was collinear (or 2 vertices were concurrent), or several faces at a vertex were dependent, or (ii) we get, implicitly, points at infinity, because the planes are parallel and the equations are inconsistent. Since realizability is really a projective property, and static rigidity is also a projective property [7], it would be natural to use projective coordinates for both the planes and the points, and remove the second defect in the choices.

In fact, we created an artificial situation by using 4 coordinates for the planes but 3 coordinates for the points. If we multiply a plane by a constant it is the same plane, so each proper realization is represented by an $f$-dimenional space of choice coordinates. If we remove these equivalent choices, and the 6-dimensional space of euclidean congruences, there are essentially $e$ choices for each polyhedron. We could eliminate this equivalence, and restore symmetry to the points and planes by insisting that no plane passes through the origin (the last coordinate is always 1). This gives a coordinate system with $c^{\prime}=3 v+3 f-i=e+6$. On the other hand we can use homogeneous projective coordinates for the points and have $4 v+4 f-i=$ $e+f+v+6$ choices and $v+f$ equivalent coordinates for the same polyhedron.

REMARK 2. Consider the set of improper realizations with all vertices as distinct points on a line, and all faces as distinct planes through this line. This allows 6 choices for the first two vertices, determining a line, 1 choice for each remaining vertex, and two choices for each face: $6+(v-2)+2 f=e+f+6$ choices. This subset of the realizations has the same dimension as the proper realizations. This shows that the variety of realizations of a spherical polyhedron is reducible. Since none of these realizations are statically rigid as bar and joint frameworks, we cannot remove the assumption of proper realizations in the theorem.

On the basis of this theorem we call an abstract polyhedron generically rigid (isostatic) if some, and therefore almost all, proper polyhedral frameworks are statically rigid (isostatic).

We must now become explicit about which discs of our bound polyhedra are to be holes, and which are to be blocks.

DEFINITION 3.12. An abstract block and hole polyhedron $(P ; B, C)$ is an abstract bounded polyhedron $(P ; D)$ with a partition of the discs $D=B \cup C$. 

$P$.

A proper block polyhedron is a proper realization $Q$ of the underlying polyhedron

A block polyhedral framework on a proper block polyhedron $((P ; B, C), Q)$ is a bar and joint framework formed by

(i) placing a joint at each vertex;

(ii) placing a bar on each boundary and each regular edge;

(iii) triangulating each regular (plane) face;

(iv) replacing each disc in the blocks $B$ by a proper block polygon (using an extra vertex for this disc).

All edges interior to a hole are omitted, so it really forms a hole in the framework. If there are no holes or blocks, this reduces to a polyhedral framework.

All of these definitions extend to refined block polyhedra, with the proviso that a refined block polyhedral framework has a refined triangulated proper plane polygon for each regular face. Theorem 3.11 also extends to these refined block polyhedra. Each refinement adds one skeletal vertex, one edge, and one dimension to the coordinate system. With the skeletal vertices placed at the end of the list, this choice is the position along the natural edge, and we maintain independence of all the linear equations.

If one block polyhedral framework on a particular proper block and hole polyhedron is statically rigid, then any other choice of triangulations and proper blocked polygons will also be statically rigid. In fact this rigidity is also preserved if we use any other isostatic framework for the discs which are blocks.

From these observations we have the following theorem.

THEOREM 3.13. The set of proper realizations of an abstract refined 3-connected spherical polyhedron is coordinatized by an open dense subset of $R^{c}$ where $c=e+f+6$. The euclidean coordinates of the points and planes are rational functions of these choice coordinates.

If one (refined) block polyhedral framework on a (refined) block polyhedron is isostatic (statically rigid), then

(i) every (refined) block polyhedral framework on the same polyhedron is isostatic (statically rigid);

(ii) almost all (refined) block polyhedral frameworks on the same abstract (refined) block and hole polyhedron are isostatic (statically rigid).

This again justifies the term generically isostatic (or generically rigid) for the abstract block and hole polyhedra fitting this theorem.

THEOREM 3.14. If an abstract block and hole polyhedron has a proper subnet $P^{\prime}$ which is generically rigid, then $P$ is generically rigid.

PROOF. The proof will proceed by induction up any construction sequence from $P^{\prime}$ to $P$.

Assume $P_{i}$ is generically statically rigid and take some statically rigid refined block polyhedral framework.

If $P_{i+1}$ comes from $P_{i}$ by edge stretching, then we have the same block polyhedral framework, so $P_{i+1}$ is also generically statically rigid.

If $P_{i+1}$ comes from $P_{i}$ by an edge split, placing $r$ on the natural edge $s t$, then we choose a distinct joint on the line of $s t$, and the vertices opposite this edge in 
the triangulations of the two faces at the edge. If we add $r$ as a 3-valent joint connected to $s$ and these select vertices, then it is not coplanar (the realization was proper) so the new framework is statically rigid. We have triangulated these two faces with triangulated refined proper polygons. This gives the required statically rigid refined block polyhedral framework for $P_{i+1}$.

Assume $P_{i+1}$ comes from $P_{i}$ by a split of face $g$ along the new edge $s, t$. In the refined block polyhedral framework for $P_{i}$ we retriangulate the face $g$ so that $s t$ is a bar. By Theorem 3.5, this gives a statically rigid framework which is isomorphic, as a graph, to a refined block polyhedral framework for $P_{i+1}$. Since $P_{i+1}$ can be realized arbitrarily near the given statically rigid realization of this graph for $P_{i}$, these nearby frameworks are also statically rigid as required.

Assume that $P_{i+1}$ comes from $P_{i}$ by pointing a boundary triangle $s, t, u$. Given the statically rigid block polyhedral framework for $P_{i}$, we add $r$ as a 3 -valent joint, off the plane of the noncollinear triangle $s, t, u$. By Theorem 3.2, this is a new statically rigid framework for $P_{i+1}$, as required.

REMARK. If we are told that $P^{\prime}$ is generically isostatic, then the proof shows that $P$ is also generically isostatic. The proof also shows that we can insert extra skeletal joints onto regular edges of any block polyhedral framework without changing the static properties.

Our major task for the rest of the paper is to find appropriate proper subnets $P^{\prime}$ for given classes of abstract block polyhedra. For example, we saw in $\S 2.3$ that every 3-connected spherical polyhedron contains a tetrahedral graph, and it is well known that a tetrahedron is isostatic unless the four vertices are coplanar.

COROLLARY 3.15. Almost all proper realizations of each abstact 3-connected spherical polyhedron are isostatic as polyhedral frameworks.

This result also follows directly from Theorem 3.12 and Alexandrov's Theorem that polyhedral frameworks on convex polyhedra are isostatic (Theorem I.3.1). This theorem was suggested by the title "Almost all simply connected surfaces are rigid" [9], but only proven there for triangulated spherical polyhedra.

\section{Cylindrical towers and 4-connectivity.}

4.1 Cylindrical towers. We have seen that a triangulated convex polyhedron is isostatic. If we remove one bar and make a quadrilateral hole, then the framework has a nontrivial infinitesimal motion in all realizations, and becomes a mechanism, almost always. It is natural to ask what new bars could return us to an isostatic framework. For example, Kuiper asked which dihedral angles (angles between adjacent faces) flex in the mechanism [14]. This is equivalent to asking which quadrilateral blocks around a dihedral angle will make a new isostatic block polyhedral framework.

There are certain obvious things to be avoided. If the quadrilateral hole and the quadrilateral block are separated by a triangle of vertices, then, in effect, the hole lives in the triangulated polyhedron one one side of this cut, and the block lives in the triangulated polyhedron on the other side. The hole side is now underbraced, and flexes, while the block side is overbraced and stressed. We show that this is all that can go wrong in a general realization. 

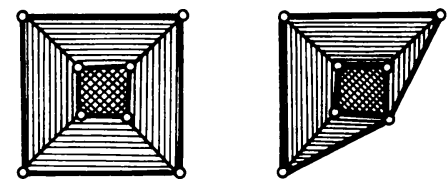

A

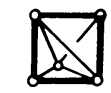

B

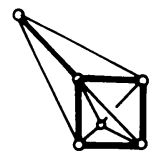

$B$
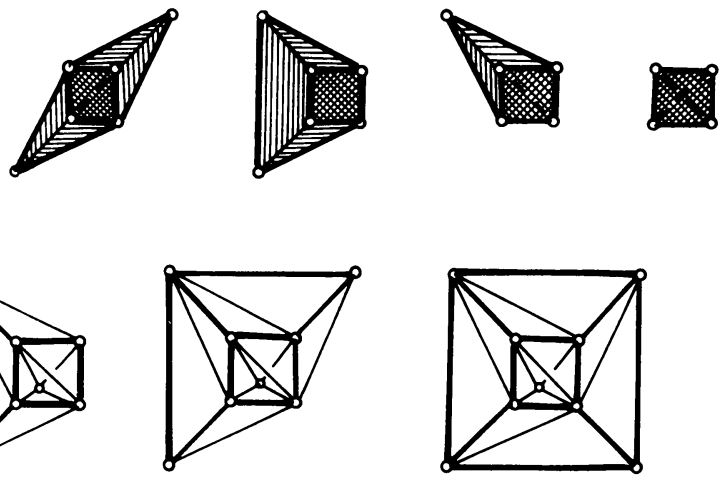

FIGURE 4.1

DEFINITION 4.1. Two discs in an abstract bound polyhedron are 4-connected if at least 4 vertices must be removed to separate all vertices of one disc from all vertices of the other.

THEOREM 4.1. An abstract block and hole polyhedron with a single quadrilateral hole and a single quadrilateral block is generically isostatic if and only if the hole and block are 4-connected.

PrOOF. Assume that the hole with vertices $a_{1}, a_{2}, a_{3}, a_{4}$ is 4-connected to the block with vertices $b_{1}, b_{2}, b_{3}, b_{4}$. By Dirac's Theorem and the 4-connectivity we have 4 vertex-disjoint paths between the discs. Up to a permutation of the labels around the hole, this means there are 4 vertex-disjoint paths $e_{1}$ from $a_{1}$ to $b_{1}, \ldots, e_{4}$ from $a_{4}$ to $b_{4}$. (Some of these may be of zero length, if the discs share vertices.) This guarantees that we have one of the quadrilateral cylinders illustrated in Figure $4.1 \mathrm{~A}$ as a proper subnet. (The blocks are shown as cross-hatched discs.) In all of these the quadrilaterial discs may be split into 2 faces, but in some they must be split, as indicated by the dotted lines, to keep the 3-connectivity (see §6). Each of these subnets can be built as an isostatic block polyhedral framework. For the first subnet in Figure 4.1 A, the verification is shown in Figure B: we begin with a block, and add a sequence of 3-valent, noncoplanar joints-ending with an example of an isostatic framework on a proper realization. A similar process verifies that all the illustrated subnets are isostatic.

Since the given abstract block polyhedron contains a generically isostatic subnet, the abstract block and hole polyhedron is also generically isostatic.

Assume that the hole and block can be separated by removing the 3 vertices $s, t, u$. For convenience, construct a convex realization of the polyhedron, and a block polyhedral framework on this realization. Each pair $s t, t u$, and $u s$ either shares an edge or a face in the polyhedron, so we can assume that each pair shares a bar in the realization. If we cut the framework into two pieces at this triangle, then one component (including $s, t, u)$ contains the block but not the hole. Therefore this component of $v^{\prime}$ vertices contains at least $3 v^{\prime}-6+1$ bar, and must contain a static stress in every realization. Since any block framework for this abstract block and hole polyhedron will contain exactly $e=3 v-6$ bars, and a static stress, it will not be statically rigid in any realization. 


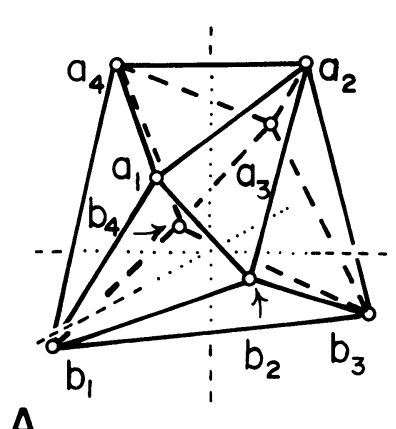

A

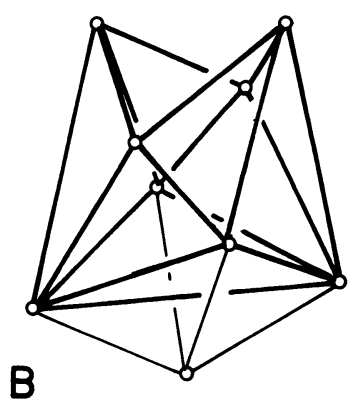

FIGURE 4.2

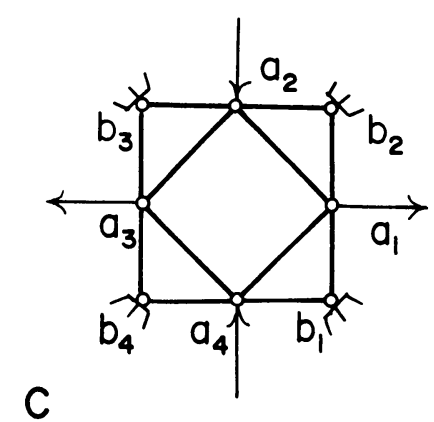

This result holds "in general". Past experience with Cauchy's theorem, and related results, shows that "general" properties may "always" happen for convex polyhedra. In this spirit we offer a simple convex counterexample.

EXAMPLE 4.1. Consider the polyhedron in Figure 4.2A-with vertices: $a_{1}=$ $(1,0,1), a_{2}=(0,1,2), a_{3}=(-1,0,1), a_{4}=(0,-1,2), b_{1}=(1,-1,-1), b_{2}=$ $(1,1,0), b_{3}=(-1,1,-1), b_{4}=(-1,-1,0)$. This is clearly convex, triangulated, and 4-connected. If we omit $a_{2} a_{4}$, to create the hole $a_{1} a_{2} a_{3} a_{4}$, and block the quadrilateral $b_{1} b_{2} b_{3} b_{4}$ with vertex $q$ and its bars (Figure B), the hole is 4-connected to the block. However, the realization given has a nontrivial infinitesimal motion: fix each $b_{i}$ with velocity $(0,0,0)$, and assign the horizontal veclocities: $v_{1}=(1,0,0)$ to $a_{1}, v_{2}=(0,-1,0)$ to $a_{2}, v_{3}=(-1,0,0)$ to $a_{3}$, and $v_{4}=(0,-1,0)$ to $a_{4}$. The view down the $z$-axis (Figure $C$ ) shows, geometrically, why these velocities are permitted, since they are perpendicular to the bars from the $b_{i}$, and they have an appropriate symmetry around the quadrilateral hole. (In [24, §I.2] we describe the general geometric conditions which make this framework infinitesimally flexible.)

For other polyhedra, the "almost always rigid" becomes "always rigid" in proper realizations. Observe that, once more, convexity is not important to the behavior.

EXAMPLE 4.2. Consider the first subnet shown in Figure 4.1A. Since this can be built as a simple framework, all proper realizations leave the required triples of bars noncoplanar-and preserve the static rigidity. Therefore all block polyhedral frameworks on proper realizations of this polyhedron are statically rigid.

The entire proof can easily be generalized to $k$-gonal holes which are $k$-connected to $k$-gonal blocks.

DEFINITION 4.2. Two discs in an abstract bound polyhedron are $k$-connected if at least $k$ vertices must be removed to separate all vertices of one disc from all vertices of the other.

THEOREM 4.2. An abstract block and hole polyhedron, with a single $k$-gonal hole and a single $k$-gonal block, is generically isostatic if and only if the hole and block are $k$-connected.

If we are satisfied with static rigidity instead of isostatic behavior, we can clearly use a larger block, which remains $k$-connected to a $k$-gonal hole. The proper subnets will be statically rigid and stressed. If the connectivity fails, the separation gives a higher count on the stresses in the overbraced component, and this will still guarantee that the structure is never statically rigid. 
THEOREM 4.2. An abstract block and hole polyhedron, with a single $k$-gonal hole and a single block is generically rigid if and only if the hole and block are $k$-connected.

4.2. 4-connected polyhedra. We have seen that the omission of one bar to create one hole and the blocking of one dihedral angle to create one block produces rigidity, provided the hole and block are disjoint and 4-connected. In particular, if the polyhedron itself is 4 -connected (i.e. deleting any 3 vertices does not disconnect the polyhedron) then any disjoint hole and block will give a generically isostatic block polyhedron. This answers the question posed by Kuiper [14, Remark 9].

THEOREM 4.3. Given a 4-connected abstract triangulated spherical polyhedron, almost all realizations as a bar and joint framework, with one bar removed, flex all the dihedral angles between the remaining triangles.

We can also imagine that the removed edge does not remove the adjacent triangles. Do the dihedral angles at these triangles also flex? The answer is yes, but a proof using bar and joint frameworks would require a number of details which we will omit here.

4.3. 5-connected spheres. There is a natural generalization suggested by Theorem 4.3. If we remove two bars from a triangulated sphere, this creates a 2-dimensional space of nontrivial motions. We say that two dihedral angles are independent in these motions if their angular velocities parameterize the space of nontrivial motions in this realization. Equivalently, bracing these two angles with blocks will remove all nontrivial infinitesimal motions.

CONJECTURE 4.1. Given any 5-connected abstract triangulated spherical polyhedron, then almost all realizations as a bar and joint framework with 2 bars removed leave each pair of dihedral angles of the remaining triangles independent.

If the two removed bars and the two chosen dihedral angles each make pentagonal discs, then the conjecture follows from Theorem 4.2. For the other cases we will need new results.

CONJECTURE 4.2. If a 5-connected abstract block and hole polyhedron has two quadrilateral holes and one pentagonal block, then the block and hole polyhedron is generically isostatic.

CONJECTURE 4.3. If a 5-connected abstract block and hole polyhedron has two quadrilateral blocks and one pentagonal hole, then the block and hole polyhedron is generically isostatic.

REMARK. By a general "reversal" property of block polyhedral frameworks, Conjectures 4.2 and 4.3 turn out to be equivalent (see $\S 6$ ).

CONJECTURE 4.4. If a 5-connected abstract block and hole polyhedron has two quadrilateral blocks and two quadrilateral holes, then the abstract block and hole polyhedron is generically isostatic.

We have proper subnets for each of the conjectures. In Figure $4.3 \mathrm{~A}$ we show a subnet for two quadrilateral holes, and one pentagonal block, in Figure B we have the reversed subnet, and in Figure $\mathrm{C}$ we show a subnet of two quadrilateral holes 

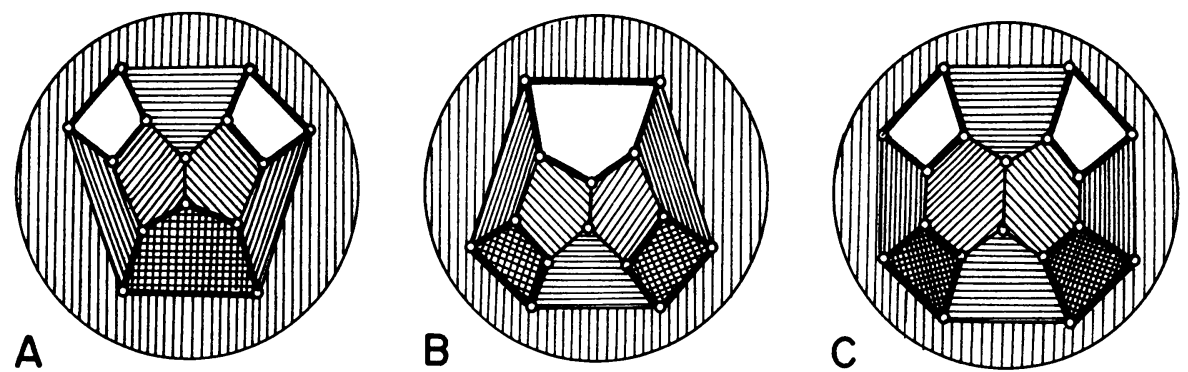

FIGURE 4.3

and two quadrilateral blocks. (To give some symmetry to the drawings the exterior circle is now a single regular face.) We conjecture that all 5-connected configurations contain one of these or a regular contraction. By a regular contraction we mean the shrinking to a vertex of some regular edges in the subnet which have one 3-valent end at a hole or block. Such a regular contracted subnet has an isostatic realization, as a block polyhedral framework, if and only if the original subnet has an isostatic realization. (For a 3-valent vertex at a hole, we simply reverse the contraction by adding the 3-valent vertex; for a 3 -valent vertex at a block, we use the reversal process mentioned above.)

Even when we cannot characterize the general arrangements which guarantee suitable initial configurations, we can use the given subnets to generate all the proper extensions: the 3-connected spherical polyhedral containing this proper subnet. Our techniques will always generate large classes of generically isostatic block polyhedral frameworks. In this spirit, we observe that Figure 2.1B shows other generically isostatic configurations which can be used as proper subnets.

\section{Related patterns and results.}

5.1 Extended polyhedral frameworks. In a variety of settings it is very useful to know about rigid bar and joint circuits: statically rigid frameworks in which the removal of any one bar leaves an isostatic framework. Such a circuit provides the protection of an optimal overbracing-one failure of a member will not cause a disaster.

Theorem 4.4 converts to a theorem about circuits. We can replace the quadrilateral block by a single tetrahedron on these four vertices. In effect this adds a single bar across a dihedral angle. The static rigidity with another bar removed, now becomes the guarantee that this extended framework is a circuit, in general realizations.

THEOREM 5.1. If an abstract triangulated spherical polyhedron is 4-connected, then adding a single bar across any dihedral angle creates a generic circuit.

PROOF. The triangulated sphere is isostatic in general (and all convex) realizations. This covers the deletion of the extra edge.

If an edge not in the tetrahedron is deleted, this leaves a quadrilateral hole and a quadrilateral block, and Theorem 4.2 guarantees this is isostatic.

If the bar is in the tetrahedron, but opposite to the added bar, then its removal also leaves a triangulated sphere. 

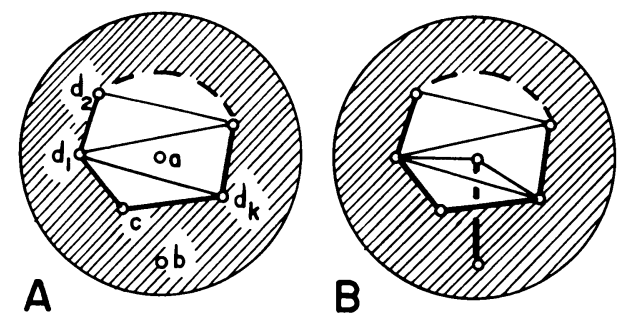

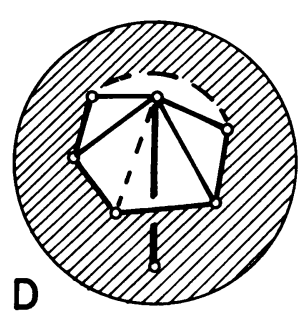

\section{FIGURE 5.1}

If the angle is $a c$ and the omitted bar is $a b$, then we proceed as follows. The polyhedron $P^{*}$, with $a$ and all adjacent edges omitted, is still 3-connected with a modified face $g: d_{1}, \ldots, d_{k}, c$. This can be realized as an isostatic polyhedral framework, with $g$ triangulated so that no extra edges enter vertex $c$. We now add the vertex $a$ in the plane of $g$, but not collinear with any two vertices (Figure 5.1A). If we connect $a$ to 2 vertices $d_{1}$ and $d_{k}$, and to $b$, this will still give an isostatic framework (Figure B). Finally, we can replace the face $g$ by all edges to $a$ which appear in $P$, except $a c$ (a retriangulation of the polydon $d_{1}, \ldots, d_{k}, a$ (Figure $\mathrm{C}$ )). This is a plane-isostatic framework, and gives a new isostatic framework for $P$. A small movement of $a$, out of the plane of $g$, yields the required isostatic proper realization (Figure D).

REMARK. Example 4.1 shows that this result only holds "in general", and does not hold for all convex realizations.

Could the extra bar be added between any two nonadjacent vertices? Yes, but we need to alter the vocabulary of $\S \S 2-4$ to apply to frameworks with special added edges and deleted edges, rather than frameworks with special faces.

DEFINITION 5.1. An abstract extended spherical polyhedron is an abstract 3connected spherical polyhedron $P$, with a set $C^{\prime}$ of edges of the polyhedron and a set $B^{\prime}$ of pairs of vertices which do not share a face in the polyhedron. A face is open if some edge is in $C^{\prime}$, otherwise it is regular.

A proper subnet of an abstract extended spherical polyhedron $\left(P ; B^{\prime}, C^{\prime}\right)$ is a proper subnet $P^{\prime}$ of $P$ which contains, as paths of length 1 all edges in $B^{\prime}, C^{\prime}$, and all edges of open faces of $\left(P, B^{\prime}, C^{\prime}\right)$.

An extended (refined) polyhedral framework for a proper subnet $P^{\prime}$ of an abstract extended polyhedron is a proper realization of the underlying polyhedron as a bar and joint framework in 3-space with

(i) a joint for each vertex;

(ii) a bar for each edge of the polyhedron not in $C^{\prime}$, and a bar for each pair in $B^{\prime}$;

(iii) additional bars for each regular face, to give a proper (refined) triangulated polygon for the face.

The previous inductions and coordinate system for block polyhedra clearly apply to the extended polyhedra. We record the essential facts.

THEOREM 5.2. If one (refined) extended polyhedral framework on a (refined) extended polyhedron is isostatic, then

(i) every (refined) extended polyhedral framework on the same polyhedron is isostatic; 


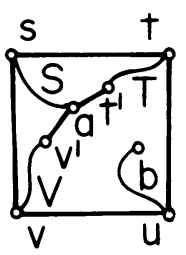

A
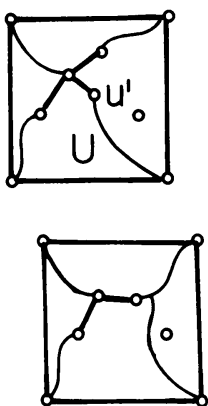

B
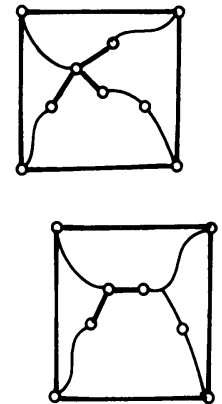

C
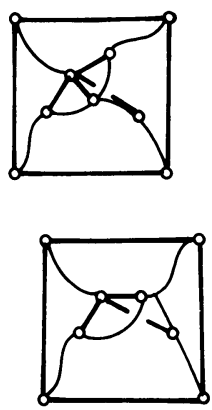

D
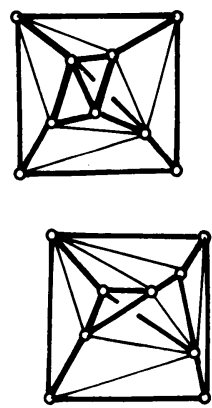

E

FIGURE 5.2

(ii) almost all (refined) extended polyhedral frameworks on the same abstract (refined) abstract extended polyhedron are isostatic.

If an abstract extended polyhedron has a proper subnet $P^{\prime}$ which is generically isostatic, then $P$ is generically isostatic.

THEOREM 5.3. If an abstract 3-connected triangulated spherical polyhedron is extended by a single bar ab between nonadjacent vertices, and the resulting graph is 4-connected, then the resulting extended polyhdedral framework is a rigid circuit in generic realizations.

ProOF. Since the original triangulated sphere gives an isostatic framework in generic realization, we know that the extended polyhedral framework is generically rigid, and that the added bar $a b$ can be omitted.

If we omit a bar $a c$ adjacent to the extending bar $a b$, then the resulting framework is isostatic.

Assume that the omitted edge $s u$ is not adjacent to the extending bar. The omission creates a quadrilateral stuv. By the 4-connectivity of the polyhedron, we can find 4 vertex disjoint paths to this quadrilateral, with 3 beginning at $a$, and 1 at $b$ (Figure 5.2A). Around the vertex $a$ there is a rim of vertices attached to $a$, forming a simple polygon. For each of the paths $T$ and $V$ we choose the last rim element $\left(t^{\prime}\right.$ and $\left.v^{\prime}\right)$ in the path, and shorten the path to $T^{\prime}=a, t^{\prime}, \ldots, t$, and $V^{\prime}: a, v^{\prime}, \ldots, v$. We connect $u$ to $a$ by a path which avoids $t, v$, and $b$ (using the 4-connectivity). After shortening this path, to have only one vertex on the rim, the net looks like Figure B, up to relettering.

We now connect the vertex $b$ to the vertex $s$ by 3 vertex-disjoint paths which avoid $a$. At most two of these can contact the rim. By simple topology around the rim, we must have two paths $X$ and $Y$ with (i) first contact on one of the paths, say $U^{\prime}$, at $x^{\prime \prime}$ and $y^{\prime \prime}$ respectively and (ii) at least one (say $X$ ) avoiding the entire rim. If $Y$ contacts $U$ before touching the rim, we insert $x^{\prime \prime}, \ldots, b, \ldots, y^{\prime \prime}$ to replace $x^{\prime \prime}, \ldots, y^{\prime \prime}$ in $U^{\prime}$, and create a subnet like Figure $\mathrm{C}$, with no rim vertices beyond $u^{\prime}$. If $Y$ contacts the rim at $y^{\prime}$, before $y^{\prime \prime}$, we insert $a, y^{\prime}, \ldots, b, \ldots, x^{\prime \prime}$ in place of $a, u^{\prime}, \ldots, x^{\prime \prime}$ at the beginning of $U^{\prime}$, and again create a subnet like Figure $\mathrm{C}$, with no rim vertices beyond $u^{\prime}$.

Finally, we add sections of the rim (which cannot cross the other existing paths) to create a subnet in Figure D, with the extending bar $a b$ included. These subnets 

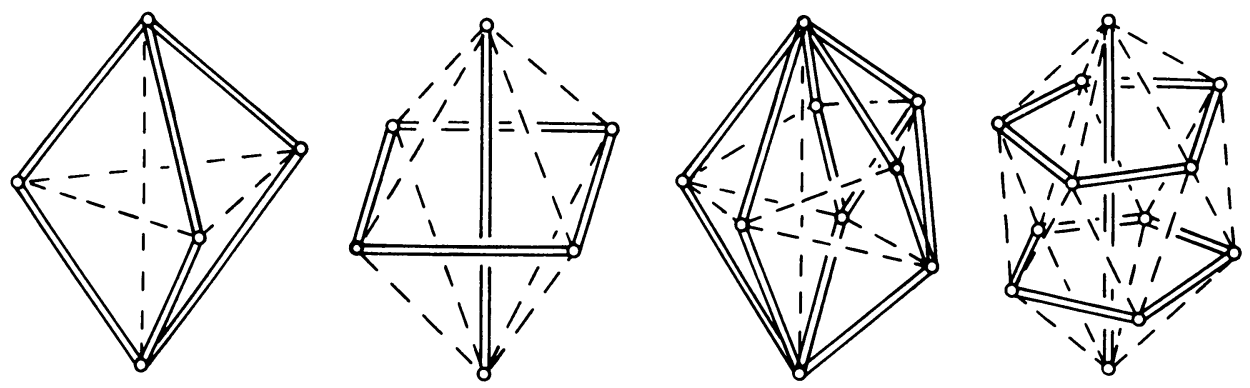

FIGURE 5.3

have isostatic realizations (Figure 5.3). As usual, some of the paths may be of zero length, within a polyhedron. However, by our assumptions, at most one path directly to $a$, or $b$, such as $U$ or $T$, could be of length zero. All the possible contracted subnets can easily be derived, and constructed as isostati: franeworks. Since all cases produce a subnet with isostatic realizations, Theorem 5.2 applied to show that $P$ minus the bar $s u$ is also generically isostatic.

Since the intersection of a finite number of open dense subsets (one for each edge of $P$ ), is also an open dense subset, we know that $P$ is a statically rigid circuit in generic realizations.

As mentioned in the Introduction, these circuits can be built as minimal statically rigid tensegrity frameworks $[17]$. Figure 5.3 shows several examples of such frameworks, with cables as dotted lines and struts as double lines.

We conclude this section with the corresponding generalization of Conjecture 4.1.

CONJECTURE 5.1. Given any triangulated sphere and any two added edges, such that the resulting graph is 5-connected, then in generic realizations any two bars can be omitted to leave an isostatic framework.

6. Conclusions. This paper has concentrated on proving that certain modified polyhedral frameworks are generically isostatic in space. There are a number of interesting geometric properties which hold for these block polyhedral frameworks:

(a) If a realization is statically rigid, then any projective equivalent realization is statically rigid.

(b) If a block polyhedral framework is stressed (infinitesimally flexible), then the reversed framework, created by switching blocks and holes, is infinitesimally flexible (stressed).

(c) If a block polyhedral framework is isostatic, then the reversed framework is also isostatic, in the same realization.

(d) If a block polyhedral framework is statically rigid, then the polar block polyhedral framework, built on the polar polyhedron, with blocks for blocks, holes for holes, vertices for faces, and faces for vertices, is also statically rigid.

These geometric results will be explored elsewhere.

Most of these results also apply to 2-connected spheres, if we make some small modifications. For example, as was noted by Barnette and Grünbaum [4], every 2-connected sphere has a Steinitz list. There is a major problem about which 2connected spheres have proper realizations, but this centers on the existence of 
appropriate subnets to start the inductions. Since our basic results were worded as a passage from an assumed subnet to a final polyhedron, they easily generalize.

DEFINITION 6.1. A proper subnet $P^{\prime}$ of a bounded 2-connected polyhedron $(P, D)$ is a subnet $P^{\prime}$ such that

(i) $P^{\prime}$ contains all vertices, edges, and faces of the disc set $D$;

(ii) the natural vertices and edges of $P^{\prime}$ form a 2-connected planar graph.

(iii) the vertices of $P$ which are not natural vertices of $P^{\prime}$ are 3-connected to the natural vertices of $P^{\prime}$.

We have a simple generalization of Theorem 2.1, which follows from the same proof.

THEOREM 6.1. Given any bounded 2-connected polyhedron $(P, D)$ and proper subnet $P^{\prime}$, there is a sequence of proper subnets $P^{\prime}=P_{1}, P_{2}, \ldots, P_{n}=P$ such that $P_{n+1}$ comes from $P_{i}$ by

Step (a) replacing a path in $P_{i}$ of one regular edge in $P$ by a longer path in $G$ joining the same terminal vertices, pairwise disjoint from the paths in $P_{i}$ (edge stretching);

Step (b) dividing a path in $P_{i}$ into two paths at a path vertex, which becomes a new skeletal vertex of $P_{n+1}$ (edge splitting);

Step $(\mathrm{c})$ inserting a new path (disjoint from the paths in $P_{i}$ ), such that the terminal vertices of the path are skeletal or natural vertices of $P_{i}$, and they are separated by the remaining natural vertices of $P_{i}$ (face splitting);

Step (d) inserting a 3-valent vertex attached to threee vertices $s, t, u$, such that all 3 edges among $s, t, u$ are boundary edges (pointing a boundary triangle).

THEOREM 6.2. Given a construction sequence $P^{\prime}=P_{1}, \ldots, P_{n}=P$ for an abstract 2-connected spherical polyhedron $P$, such that $P^{\prime}$ has a proper realization, then each refined abstract spherical polyhedron $P_{i}$ has a proper realization.

The block polyhedral frameworks are defined as before.

THEOREM 6.3. The set of proper realizations of an abstract 2-connected spherical polyhedron is coordinatized by an open dense subset of $R^{c}$ where $c=e+f+6$. The euclidean coordinates of the points and planes are rational functions of these choice coordinates.

If one block polyhedral framework on $P$ is statically rigid, then the statically rigid realizations form an open dense subset in this coordinatization.

Again the term generically isostatic (or generically rigid) is used for the abstract block and hole polyhedra fitting this theorem.

THEOREM 6.4. If an abstract block and hole 2-connected polyhedron has a proper subnet $P^{\prime}$, which is generically rigid, then $P$ is generically rigid.

THEOREM 6.5. An abstract 2-connected block and hole polyhedron, with a single $k$-gonal hole and a single $k$-gonal block, is generically isostatic if and only if the hole and block are $k$-connected, and the graph obtained by adding the block vertices and its edges is 3-connected.

If we look back at Figure 4.1A, we see, for example, that this theorem allows us to not subdivide any of the holes or blocks. 
The techniques employed here will not apply directly to polyhedra, and polyhedra frameworks built around other surfaces. For example, for toroidal surfaces we may not even have a Steinitz list of the vertices and faces, and there remain unsolved problems about the realizability of even well-connected abstract toroidal polyhedra. Nevertheless, we believe that the analogues of many of these results will be true for properly realized toroidal polyhedra. The techniques developed here can be combined with other inductive techniques for triangulated surfaces to show that each triangulated one-holed torus is generically 3-rigid. A more general vertex splitting technique [29] has recently been applied by Allan Fogelsanger to prove the generic rigidity of all triangulated closed surfaces in 3-space.

In another direction, the results of this paper and the first paper are simple cases of the determination problem for spherical polyhedra: which sets of dataedge lengths, dihedral angles, coplanarity, etc.-will make a spatial polyhedron unique, at least locally? The answer is critical to providing minimal, sufficient descriptions, and maximal, independent choices for polyhedra in computer aided design and computer reconstruction of spatial scenes. Some initial results, including the translation of the theorems given here, will be presented in [28].

The study of these questions requires a continuing interaction of the practical algorithmic questions, and the general geometric and combinatorial analysis of polyhedra. In such an interaction, the results will flow in both directions. Our two papers have clearly been built on the classical theorems of Cauchy and Steinitz on convex polyhedra. Conversely, the $d$-dimensional analogue of Alexandrov's Theorem, on the rigidity of convex $d$-polytopes with triangulated 2 -faces, gives a new, stronger form of the lower bound theorem for the number of edges in a general $d$ polytope $[3, \mathbf{1 3}]$. We will also see, in [28], that a classical theorem of Clerk Maxwell about the statics of plane frameworks gives important insight into the structure of dihedral angles in a spatial polyhedron.

The current developments in discrete applied geometry should be fruitful for both the mathematicians, and the applied workers.

\section{REFERENCES}

1. A. D. Alexandrov, Konvex polyeder, German translation, Akademie-Verlag, Berlin 1958.

2. L. Asimov and B. Roth, The rigidity of graphs, Trans. Amer. Math. Soc. 245 (1978), $279-289$.

3. D. Barnette, A proof of the lower bound conjecture, Pacific J. Math. 46 (1973), 349-354.

4. D. Barnette and B. Grünbaum, On Steinitz's theorem concerning 3-polytopes and some properties of planar graphs, The Many Facets of Graph Theory, Lecture Notes in Math., vol. 110, Springer-Verlag, Berlin and New York, 1969, pp. 27-40.

5. R. Connelly, Rigidity of polyhedral surfaces, Math. Mag. 52 (1979), 275-283.

6. _ The rigidity of certain cabled framewororks and the second order rigidity of arbitrarily triangulated convex surfaces, Adv. in Math. 37 (1980), 272-298.

7. H. Crapo and W. Whiteley, Statics of frameworks and motions of panel structures: a projective geometric introduction, Structural Topology 6 (1982), 43-82.

8. G. A. Dirac, Extensions of Menger's theorem, J. London Math. Soc. 38 (1963), 146-163.

9. H. Gluck, Almost all simply connected surfaces are rigid, Geometric Topology, Lecture Notes in Math., vol. 438, Springer-Verlag, Berlin and New York, 1975, pp. 225-239.

10. J. Graver, A combinatorial approach to infinitesimal rigidity, preprint, Syracuse Univ., Syracuse, New York, 1984.

11. B. Grünbaum, Convex polytopes, Wiley, New York, 1968.

12. _ Lectures in lost mathematics, mimeograph notes, Univ. of Washington, Seattle, Washington, 1976. 
13. G. Kalai, Rigidity and the lower bound theorem. I, Invent. Math. 88 (1987), 125-151.

14. N. Kuiper, Spheres polyedriques flexible dans $E^{3}$, d'apres Robert Connelly, Séminaire Bourbaki 1977/78, Lecture Notes in Math., vol. 710, Springer-Verlag, Berlin and New York, 1979, pp. 147-168.

15. G. Laman, On graphs and the rigidity of plane skeletal structures, J. Engineering Math. 4 (1970), 331-340.

16. L. A. Lyusternik, Convex figures and polyhedra (Russian), Moscow, 1956; English transl., Dover, New York, 1963.

17. B. Roth and W. Whiteley, Tensegrity frameworks, Trans. Amer. Math. Soc. 265 (1981), 419446.

18. G. T. Salee, Incidence graphs of convex polytopes, J. Combin. Theory 2 (1967), 466-506.

19. E. Steinitz and H. Rademacher, Vorlesungen über die Theorie der Polyeder, Springer-Verlag, Berlin and New York, 1934.

20. T. Tarnoi, Simultaneous static and kinematic indeterminacy of space trusses with cyclic symmetry, Internat. J. Solids Struct. 16 (1980), 345-356.

21. T-S. Tay and W. Whiteley, Recent advances in the generic rigidity of frameworks, Structural Topology 9 31-38.

22. _ـ Generating isostatic frameworks, Structural Topology 11 (1985), 21-69.

23. N. White and W. Whiteley, The algebraic geometry of stresses in frameworks, SIAM J. Algebraic Discrete Method 4 (1983), 481-511.

24. W. Whiteley, Introduction to structural geometry. I, II, notes, Champlain Regional College, Quebec, Canada, 1976.

25. _ Infinitesimally rigid polyhedra. I: Statics of frameworks, Trans. Amer. Math. Soc. 285 (1984), 431-465.

26. __ Realizability of polyhedra, Structural Topology 1 (1979), 46-58.

27. __ Rigidity and polarity. I: Statics of sheetworks, Geom. Dedicata 22 (1987), 329-362.

28. _ Determination of spherical polyhedra (to appear).

29. __ Vertex splitting in isostatic frameworks, Structural Topology (to appear).

30. H. Whitney, Non-separable and planar graphs, Trans. Amer. Math. Soc. 34 (1932), 339-362.

31. _ 2-isomorphic graphs, Amer. J. Math. 55 (1933), 245-254.

Department of Mathematics, Champlain Regional College, St. Lambert, QUEBEC J4P-3P2, CANADA 